\title{
FINITE REFLECTION GROUPS
}

\author{
BY \\ ROBERT STEINBERG
}

1. Introduction. The finite groups generated by reflections (g.g.r.) of real Euclidean space of $n$ dimensions $\left(E^{n}\right)$ have been classified by Coxeter [4]. He has noticed a number of properties common to these groups, but has been able to prove them only by verification in the individual cases. Our prime purpose here is to give general proofs of some of these results (1.1 to 1.4 below).

If $B$ is a finite g.g.r. on $E^{n}$, the reflecting hyperplanes (r.h.) all pass through one point, which may be taken as the origin 0 , and partition $E^{n}$ into a number of chambers each of which is a fundamental region of $(5)$; further $(B)$ is generated by the reflections in the walls of any one of these chambers. The group (B) is irreducible in the usual algebraic sense if and only if there are $n$ linearly independent r.h. and there is no partition of the r.h. into two nonempty sets which are orthogonal to each other $[7, \mathrm{p} .403]$. In this case each chamber is a simplicial cone with vertex at $0[3$, p. $254 ; 4$, p. 590].

This leads us to the first result of Coxeter [4, p. 610]:

1.1. Theorem. If Bf is a finite irreducible g.g.r. on $E^{n}$ and if $h$ is the order of the product of the reflections in the walls of one of the fundamental chambers, then the number of reflecting hyperplanes is $n h / 2$.

Associated with each simple Lie algebra (or Lie group) of rank $n$ over the complex field there is a finite irreducible g.g.r. (5) on $E^{n}$ and a set of vectors (roots) normal to the corresponding r.h. $[1 ; 13]$. There then exists a fundamental set of roots and a so-called dominant root relative to this set (definitions in $\S \S 6$ and 8).

Then Coxeter's second observation $[6$, p. 234] is this:

1.2. TheOREM. If $\alpha_{1}, \alpha_{2}, \cdots, \alpha_{n}$ is a fundamental set of roots for a simple Lie algebra of rank $n$, and if $\sum y^{j} \alpha$, is the dominant root, then the number of reflecting hyperplanes of the corresponding group (\$) (or one-half the number of roots) is $n\left(1+\sum y^{j}\right) / 2$.

From 1.2 (see [6, p. 212]) one immediately gets:

1.3. Theorem. The dimension of the Lie algebra (or Lie group) is $n\left(2+\sum y^{j}\right)$.

As Coxeter [6, p. 212] has remarked, this is an interesting analogue to the formula of Weyl for the order of (5), namely, $g=f \cdot n ! \Pi y^{j}$, with $f-1$ denoting the number of $y$ 's equal to 1 .

Received by the editors, October 19, 1957. 
The proof of 1.2 (and hence also of 1.3) is rather short and is independent of 1.1. If 1.1 and 1.2 are combined, the result is the formula [6, p. 234]:

\subsection{Theorem. $\sum y^{j}=h-1$.}

Following the proof of 1.1, 1.2 and some related results concerning root systems and Petrie polygons, in the last section there is set forth another curious property of root systems which the author has recently discovered but has been unable to prove by general methods.

The abbreviations g.g.r. and r.h. introduced above are used throughout the paper.

2. Preliminary lemmas. Recall that a tree is a finite connected graph with no circuits and that an end node is one linked to at most one other node.

2.1. Lemma. A tree has an end node.

2.2. Lemma. If each circuit of a graph has even length, then the nodes can be placed in two classes so that each link joins two nodes belonging to distinct classes.

2.3. Lemma. Let $p_{1}, p_{2}, \cdots, p_{n}$ be the nodes of a tree $T$. Then any circular arrangement of the numbers $1,2, \cdots, n$ can be obtained from any given one by a sequence of moves each of which consists of the interchange of a pair ij which satisfy the condition that $i$ and $j$ are adjacent on the circle and $p_{i}$ and $p_{j}$ are not directly linked on $T$.

Proof. See $[10$, pp. 49,151$]$ for proofs of the first two results. Since 2.3 (cf. [4, p. 602]) is clearly true for $n=1$ or 2 , we assume $n \geqq 3$ and proceed by induction. By 2.1 there is a node $p_{n}$ joined to exactly one other node of $T$. If $p_{i}$ and $p_{j}$ are nodes other than $p_{n}$, at least one of them, say $p_{i}$, is not linked to $p_{n}$. Thus if the numbers $i n j$ occur in this order on the circle, and if $p_{i}$ and $p_{j}$ are not linked, the following moves can be made: $i n j \rightarrow n i j \rightarrow n j i$; here $i$ and $j$ have been interchanged in the circular arrangement restricted to $1,2, \cdots$, $n-1$. Thus by the inductive hypothesis applied to $T-p_{n}$ one can transform the numbers $1,2, \cdots, n-1$ into any given arrangement, and one can then move the number $n$ around the circle to its required position.

As is customary, we associate with each spherical $(n-1)$-simplex (or each simplicial cone in $E^{n}$ ) a graph by choosing a node for each wall of the simplex (or cone) and linking two nodes if and only if the corresponding walls are not orthogonal. One can also associate a graph with a symmetric $n \times n$ matrix $\left(a_{i j}\right)$ by introducing $n$ nodes $p_{1}, \cdots, p_{n}$ and linking $p_{i}$ and $p_{j}$ if and only if $a_{i j} \neq 0$.

2.4. Lemma. Let $F$ be a spherical ( $n-1)$-simplex on a sphere of radius 1. Assume that the interior dihedral angles of $F$ are nonobtuse and that the graph 
corresponding to $F$ is connected. Then the spherical distance between any two points of $F$ is less than $\pi / 2$.

This result can easily be proved by the methods used by Cartan [3, p. 253] to establish similar results.

2.5. Lemma. If $\left(a_{i j}\right)$ is a symmetric matrix containing only non-negative real entries and if the corresponding graph is connected, then $\left(a_{i j}\right)$ has a characteristic value which is positive, strictly larger than all other characteristic values, and such that the corresponding characteristic vector can be chosen to have only positive entries.

This is a special case of a theorem of Frobenius [9, p. 471].

3 . Spherical simplexes. Throughout this section the following conventions and assumptions are made. Let $F$ denote a spherical simplex on the unit sphere in $E^{n}$ such that the corresponding graph is connected and has only even circuits. By 2.2 the walls of $F$ can be so labeled that

3.1. $W_{1}, W_{2}, \cdots, W_{s}$ are mutually orthogonal as also are $W_{s+1}, \cdots, W_{n}$.

Set $E^{(2)}=W_{1} \cdot W_{2} \cdots W_{s}$ and $E^{(1)}=W_{s+1} \cdots W_{n}$, these being "opposite edges" of $F$. In the space $E^{n}$ in which $F$ is embedded let $\epsilon_{1}, \epsilon_{2}, \cdots, \epsilon_{n}$ be inwardly directed unit normals to $W_{1}, W_{2}, \cdots, W_{n}$, and let $\epsilon^{1}, \epsilon^{2}, \cdots, \epsilon^{n}$ be the dual basis, so that $\left(\epsilon_{i}, \epsilon^{j}\right)=\delta_{l}^{j}$, where $(\cdot, \cdot)$ denotes the inner product of $E^{n}$. Let $G=\left(g_{i j}\right)=\left(\left(\boldsymbol{\epsilon}_{i}, \boldsymbol{\epsilon}_{j}\right)\right)$, so that $G^{-1}=\left(g^{i j}\right)=\left(\left(\boldsymbol{\epsilon}^{i}, \boldsymbol{\epsilon}^{j}\right)\right)$. Corresponding to each characteristic value $x$ of $1-G$ we introduce a characteristic vector $\left(l_{1}, l_{2}, \cdots, l_{n}\right)$ and the notations

$\bar{\sigma}=\sum_{1}^{s} l_{i} \epsilon^{i}, \bar{\tau}=\sum_{s+1}^{n} l_{i} \epsilon^{i}, \bar{\rho}=\bar{\sigma}+\bar{\tau}, \sigma=\bar{\sigma} /(\bar{\sigma}, \bar{\sigma})^{1 / 2}, \tau=\bar{\tau} /(\bar{\tau}, \bar{\tau})^{1 / 2}, \rho=\bar{\rho} /(\bar{\rho}, \bar{\rho})^{1 / 2}$.

3.2. Lemma. If the assumptions of the preceding paragraph are made, then (1) corresponding to the characteristic value $x=0$ of $1-G$ the point $\sigma$ is orthogonal to the edge $E^{(2)}$, and $\tau$ is orthogonal to $E^{(1)} ;(2)$ corresponding to each $x \neq 0$ the spherical line joining $\sigma$ and $\tau$ cuts the edges $E^{(1)}$ and $E^{(2)}$ orthogonally, has $\rho$ as one of its mid-points, and is of length $\cos ^{-1} x$; (3) the largest characteristic value $x$ is positive and has multiplicity 1 , the corresponding l's may be taken as positive, so that one of the open segments $\sigma \tau$ is complctely interior to $F$, and the length $\sigma \tau=\cos ^{-1} x$ in this case is the absolutely minimum distance between $E^{(1)}$ and $E^{(2)}$.

Proof. Because of 3.1 the matrix $G$ can be written in partitioned form

$$
G=\left(\begin{array}{ll}
1 & A \\
A^{\prime} & 1
\end{array}\right)
$$

with the 1's denoting identity matrices and $A^{\prime}$ the transpose of $A$. Let 


$$
G^{-1}=\left(\begin{array}{ll}
B & C \\
C^{\prime} & D
\end{array}\right)
$$

be the corresponding form of $G^{-1}$. Then $G^{-1} \cdot G=1$ implies
3.3
$B A+C=0$,
$C^{\prime}+D A^{\prime}=0$.

Let $l^{(1)}$ and $l^{(2)}$ be the column vectors with co-ordinates $l_{1}, l_{2}, \cdots, l_{s}$ and $l_{s+1}, \cdots, l_{n}$ respectively. Then since $\left(l_{1}, l_{2}, \cdots, l_{n}\right)$ and $x$ are corresponding characteristic vector and value of $1-G$, we have
3.4
$x l^{(1)}+A l^{(2)}=0$,
$A^{\prime} l^{(1)}+x l^{(2)}=0$.

Because of 3.3 these equations yield:

$$
3.5 \quad x B l^{(1)}-C l^{(2)}=0, \quad C^{\prime} l^{(1)}-x D l^{(2)}=0 \text {. }
$$

If $x=0$, the equation $C^{\prime} l^{(1)}=0$ in co-ordinate form reads

$$
0=\sum_{j=1}^{8} g^{i j} l_{j}=\left(\epsilon^{i}, \sum_{j=1}^{s} l_{j} \epsilon^{j}\right)=\left(\epsilon^{i}, \bar{\sigma}\right), \quad i=s+1, \cdots, n,
$$

so that $\bar{\sigma}$ (or $\sigma$ ) is orthogonal to $E^{(2)}$; similarly $\tau$ is orthogonal to $E^{(1)}$. Next suppose $x \neq 0$. Now 3.5 yields $l^{(1)^{\prime}} B l^{(1)}=l^{(2) \prime} D l^{(2)}$, or $\sum \sum_{1}^{s} g^{i j} l_{i} l_{j}$ $=\sum \sum_{s+1}^{n} g^{i j} l_{i} l_{j}$, or $(\bar{\sigma}, \bar{\sigma})=(\bar{\tau}, \bar{\tau})$. Thus $\rho$ is one of the mid-points of $\sigma \tau$. We can normalize the $l$ 's so that

$$
(\bar{\sigma}, \bar{\sigma})=(\bar{\tau}, \bar{\tau})=1, \quad \bar{\sigma}=\sigma, \quad \bar{\tau}=\tau .
$$

Then the equations 3.5 say that the vectors $x \sigma-\tau$ and $\sigma-x \tau$ are respectively orthogonal to $E^{(1)}$ and $E^{(2)}$. Thus the line $\sigma \tau$ cuts $E^{(1)}$ and $E^{(2)}$ orthogonally. Another application of 3.5 yields $\cos \sigma \tau=l^{(1) \prime} C l^{(2)}=x l^{(1)^{\prime}} B l^{(1)}=x$. Finally suppose that $x$ is the largest characteristic value of $1-G$. Since $F$ has no obtuse dihedral angles, the entries of $1-G$ are non-negative; since the graph of $F$ is connected, so also is the graph of $1-G$. Hence 2.5 implies that $x$ is positive and of multiplicity 1 , and that the l's can be chosen all positive. It is easily seen that the problem of finding the minimum distance from a point $\sigma$ $=\sum_{1}^{s} l_{i} \epsilon^{i}$ of $E^{(1)}$ to a point $\tau=\sum_{s+1}^{n} l_{i} \epsilon^{i}$ of $E^{(2)}$, because of the restriction $(\sigma, \sigma)=(\tau, \tau)=1$, gives Lagrange equations which lead to 3.5. Thus the minimum distance $\cos ^{-1} x$ corresponds to the largest value of $x$, and 3.2 is proved.

From 3.2 we easily deduce:

3.6. LemmA. In addition to the assumptions made in 3.1 , let $R_{1}, R_{2}, \cdots, R_{n}$ denote the reflections in the walls $W_{1}, W_{2}, \cdots, W_{n}$, and set $S=R_{1} R_{2} \cdots R_{s}$, $T=R_{s+1} \cdots R_{n}$ and $R=S T$ (operations performed from right to left). Then $R$ is a product of translations along the lines $\sigma \tau$ of 3.2 through distances of $2 \sigma \tau$ $=2 \cos ^{-1} x$ corresponding to nonzero values of $x$ together with the central inversion in the space spanned by the $\sigma$ 's and $\tau$ 's corresponding to $x=0$. 
Proof. By 3.1, $S$ leaves each point of $E^{(2)}$ fixed and maps each point orthogonal to $E^{(2)}$ on its antipode. Hence if $x$ is a nonzero characteristic value of $1-G$ and if $L=\sigma \tau$ is the corresponding spherical line given by 3.2, the restriction of $S$ to $L$ is equal to the reflection in $\tau$; similarly $T$ effects the reflection in $\sigma$ and $R=S T$ the translation of length $2 \sigma \tau$ along $L$. The case $x=0$ is treated similarly.

In regard to the results above, it is to be noted that Coxeter $[5 ; 8$, p. 766], by a somewhat different method and under the more restrictive assumption that the graph of $F$ is a tree, proved that the characteristic values of $1-G$ give the lengths of the basic translations of which $R$ is a product according to 3.6. The present development, which also gives a geometric interpretation to the characteristic vectors of $1-G$, was inspired by a remark of Coxeter [6, p. 233].

4. Finite irreducible g.g.r. Assume that $B$ is a finite irreducible g.g.r. leaving 0 fixed, that $F$ is a fundamental chamber, and that $F$ also denotes the corresponding spherical simplex on the unit sphere with center at 0 . Then the graph of $F$ is a tree [6, p. 195]; and, since reflections in orthogonal hyperplanes are commutative, 2.3 implies the following result due to Coxeter $[4$, p. 602]:

4.1. The products of the reflections in the $n$ walls of a fundamental chamber of a finite irreducible g.g.r. taken in the various orders are all conjugate.

Since also the dihedral angles of $F$ are submultiples of $\pi$, the various notations, assumptions (in particular 3.1), and conclusions of $\$ 3$ are applicable and will be used henceforth to refer to this specific situation.

It is now easy to prove the first main result stated in $\$ 1$ :

4.2. Theorem. If the product of the reflections in the walls of $F$ has order $h$, then the number of reflecting hyperplanes is $n h / 2$.

Proof. Let $L=\sigma \tau$ be the line corresponding to the largest characteristic value of $1-G$ as given by 3.2 , and let $R=S T$ be the product of the reflections in the walls of $F$ as in 3.6. Let $\mathfrak{S}$ be the restriction to $L$ of the group generated by $S$ and $T$; that is, $\mathfrak{S}$ is the group generated by reflections in the points $\sigma$ and $\tau$ of $L$. Then $R$ also has order $h$ in $\mathfrak{S}$ : if $R^{k}$ leaves $L$ fixed pointwise, it leaves fixed points interior to $F$ by 3.2 , and so is the identity in $(5)$. Thus $\mathfrak{F}$ has order $2 h$. Since $\mathfrak{S}$ contains the reflections in $\sigma$ and in $\tau$, and since the open segment $\sigma \tau$ is interior to $F$, it follows that $\sigma \tau$ is a fundamental region for $\mathfrak{S}$. Hence $\sigma \tau=\pi / h$ and there are $h$ transforms of each of the points $\sigma$ and $\tau$ alternating around $L$. Clearly $L$ meets r.h. at these points only; it meets $n-s$ r.h. at each transform of $\sigma$ and $s$ r.h. at each transform of $\tau$; however, each hyperplane is met twice at antipodes. Hence the number of r.h. is $n h / 2$, and 4.2 is proved.

In the following corollaries, the same assumptions as in 4.2 are made. 
4.3. Corollary. The minimum distance between $E^{(1)}$ and $E^{(2)}$ is $\pi / h$; all other extreme distances between $E^{(1)}$ and $E^{(2)}$ are integral multiples of $\pi / h$. One of the characteristics values of $R$ is $\exp (2 \pi i / h)$; all other characteristics values are integral powers of it.

Because of what has already been said, this is clear (cf. [6, p. 233]).

4.4. Corollary. If $h$ is odd, then $s=n-s$ and $W_{1}, \cdots, W_{s}$ are mapped onto $W_{s+1}, \cdots, W_{n}$ by an element of (5).

Proof. Indeed in this case $R^{(h-1) / 2}$ maps $\tau$ onto $-\sigma$ and the hyperplanes meeting at $\tau$ onto those meeting at $-\sigma$ (or at $\sigma$ ).

4.5. Corollary. If (5) contains the central inversion $I$, then $h$ is even and $I=R^{h / 2}$.

Proof. If it exists, $I$ maps $F$ onto $-F$. If $h$ is odd, the transformation $U=R^{(h-1) / 2} S$ maps $\sigma$ and $\tau$ onto $-\tau$ and $-\sigma$ respectively and hence $F$ onto $-F$ since the segment $\sigma \tau$ has points interior to $F$; clearly $U \neq I$. If $h$ is even, one sees similarly that $R^{h / 2}$ maps $F$ on $-F$ and hence is the central inversion if it exists.

This result also has been verified by Coxeter [4, p. 606]. By 4.1 it remains valid if $R_{1}, R_{2}, \cdots, R_{n}$ are multiplied in an arbitrary order to give $R$.

4.6. Corollary. Assume again the order 3.1 for the walls of $F$. Define $W_{k}=W_{j}$ and $R_{k}=R_{j}$ if $k \equiv j(\bmod n)$. Then the r.h. are

4.7

$$
R_{1} R_{2} \cdots R_{k-1} W_{k}
$$

and the reflections of (B) are

4.8

$$
R_{1} R_{2} \cdots R_{k-1} R_{k} R_{k-1} \cdots R_{1}
$$

for $k=1,2, \cdots, n h / 2$.

Proof. If $1 \leqq k \leqq s$, then $R_{1} R_{2} \cdots R_{k-1} W_{k}=W_{k}$, so that the first $s$ hyperplanes of 4.7 are those met by the line $L$ of 4.2 at the point $\tau$. Similarly one sees that the r.h. of 4.7 are listed in the order in which they are met in a trip along $L$ for a point interior to $F$ to its antipode in $-F$. Clearly 4.8 is the reflection in 4.7 .

5. Petrie polygons. Here we assume $n \geqq 3$ and consider the spherical honeycomb $C$ in which the unit sphere is cut by the r.h. This consists of a number of spherical $(n-1)$-simplexes bounded by simplexes of lower orders. In this case the following definition of a Petrie polygon (p.p.) exists (cf. $[6$, p. 223]): any $n$ consecutive vertices of a p.p. belong to one simplex of $C$, but no $n+1$ consecutive vertices do.

It easily follows that any $n$ consecutive vertices of a p.p. are distinct, that any ordered set of $n$ vertices of a simplex of $C$ determines a unique p.p., that $n-1$ ordered vertices of a simplex of $C$ determine two p.p., and 
that, if $\epsilon^{1}, \epsilon^{2}, \cdots, \epsilon^{n+1}$ are $n+1$ consecutive vertices of a p.p., then $\epsilon^{n+1}$ is the reflection of $\epsilon^{1}$ in the hyperplane determined by $\epsilon^{2}, \epsilon^{3}, \cdots, \epsilon^{n}$.

5.1. Theorem. Let $W_{1}, W_{2}, \cdots, W_{n}$ be the walls of $F$ taken in an arbitrary order, and let $\epsilon^{1}, \epsilon^{2}, \cdots, \epsilon^{n}$ be the respectively opposite vertices. Set $R_{k}=R_{j}$ and $\epsilon^{k}=\epsilon^{j}$ if $k \equiv j(\bmod n)$. Let $P$ be the Petrie polygon of which $\epsilon^{1}, \epsilon^{2}, \cdots, \epsilon^{n}$ are the first $n$ vertices. Then (1) the vertices of $P$ in order are given by $\beta^{k}=R_{1} R_{2} \ldots$ $R_{k-1} \epsilon^{k}, k=1,2, \cdots, n h ;(2)$ the vertices of $P$ are distinct; (3) the $n h(n-2)$ simplexes determined by sets of $n-1$ consecutive vertices of $P$ lie 2 in each of the $n h / 2 r . h$.

Proof. Since $R_{j} \epsilon^{k}=\epsilon^{k}$ if $j \not \equiv k(\bmod n)$, we see that $\beta^{k}=\epsilon^{k}$ for $1 \leqq k \leqq n$. Also $\left(\beta^{i}, \beta^{i+1}, \cdots, \beta^{j+n-1}\right)=R_{1} R_{2} \cdots R_{j-1}\left(\epsilon^{j}, \cdots, \epsilon^{j+n-1}\right)$, so that these $n$ consecutive $\beta$ 's are the vertices of the simplex $R_{1} R_{2} \cdots R_{j-1} F$. But $\beta^{j+n}$ can not be a vertex of this simplex since $R_{1} R_{2} \cdots R_{j-1} F \neq R_{1} R_{2} \cdots R_{j} F$. Hence (1) is proved.

Next (2) and (3) are proved under the assumption that the $W$ 's are ordered as in 3.1. If $k \neq j(\bmod n)$, the points $\beta^{k}$ and $\beta^{i}$ are distinct since they are transforms of distinct points of $F$. Thus if $\beta^{k}=\beta^{j}$, we can normalize so that $1 \leqq j \leqq n, k=j+r n, 0 \leqq r<h$; then $R^{r} \beta^{j}=\beta^{k}=\beta^{j}$. If $\beta^{j}$ lies on the line $L$ of 4.2 , then $r=0$ and $k=j$ since $R$ effects a translation of order $h$ along $L$. If $\beta^{j}$ does not lie on $L$, then either $\beta^{j} \sigma$ or $\beta^{j} \tau$ is orthogonal to $L$; say the first is and set $\sigma^{\prime}=R^{r} \sigma$. Then $\beta^{i} \sigma^{\prime}$ is also orthogonal to $L$. But $\beta^{i} \sigma^{\prime}=\beta^{i} \sigma<\pi / 2$ by 2.4 . Hence $\sigma=\sigma^{\prime}=R^{r} \sigma$ from which we conclude $r=0$ and $k=j$ as before. Since $\epsilon^{k+1}, \epsilon^{k+2}, \cdots, \epsilon^{k+n-1}$ lie on the wall $W_{k}$ of $F$, it follows on application of $R_{1} R_{2} \cdots R_{k-1}$ that $\beta^{k+1}, \cdots, \beta^{k+n-1}$ lie on the hyperplane $R_{1} R_{2} \cdots R_{k-1} W_{k}$. Then a slight modification of 4.6 yields ( 3 ).

The first step in removing the restriction on the order of the $W$ 's is to modify the order adopted in the previous paragraph by a simple cyclic permutation thus: set $W_{1}^{\prime}=W_{2}, W_{2}^{\prime}=W_{3}, \cdots, W_{n}^{\prime}=W_{1}$ and define corresponding $R^{\prime}, \epsilon^{\prime}$ and $\beta^{\prime}$. Then $\beta^{k^{\prime}}=R_{1}^{\prime} R_{2}^{\prime} \cdots R_{k-1}^{\prime} \epsilon^{k^{\prime}}=R_{2} R_{3} \cdots R_{k} \epsilon^{k+1}$ $=R_{1} \beta^{k}$ for each $k$, so that (2) and (3) are still valid for the new order. Next suppose that we have an order for which (2) and (3) hold and that two consecutive $W^{\prime}$ 's, say $W_{1}$ and $W_{2}$, are orthogonal so that $R_{1}$ and $R_{2}$ commute. Adopting the new order $W_{1}^{\prime}=W_{2}, W_{2}^{\prime}=W_{1}, W_{3}^{\prime}=W_{3}, \cdots, W_{n}^{\prime}=W_{n}$, one sees that $\beta^{j^{\prime}}=\beta^{j+1}$ and $\beta^{j+1^{\prime}}=\beta^{j}$ if $j \equiv 1(\bmod n)$ while $\beta^{j^{\prime}}=\beta^{j}$ if $j \neq 1,2(\bmod n)$. The hyperplanes determined by sets of $n-1$ consecutive $\beta$ 's are permuted in a similar manner. Thus (2) and (3) hold for the new order of the $W$ 's, and, because of 2.3 , for an arbitrary order.

This result and the following corollary are suggested by Coxeter $[6$, p. 231], but with a general proof only in the case $n=3$.

5.2. Corollary. Let $g$ be the order of (5). Then the number of $(n-2)$ simplexes in cach hyperplane of the honeycomb $C$ is $g / h$. 
Proof. There are $n ! / 2$ p.p. which contain the vertices of a given simplex, say $F$, as $n$ consecutive vertices. Since each p.p. determines $n h$ simplexes by its sets of $n$ consecutive vertices, the number of p.p. is $g \cdot(n-1) ! / 2 h$. By 5.1 each p.p. leads to $n h(n-2)$-simplexes which lie in pairs in the $n h / 2$ hyperplanes. Since each $(n-2)$-simplex is counted $(n-1)$ ! times in this enumeration, the number of $(n-2)$-simplexes in each hyperplane is

$$
(g \cdot(n-1) ! / 2 h) 2 /(n-1) ! \text { or } g / h \text {. }
$$

Coxeter has shown that the symmetry group $\&$ of each regular polytope $\Pi$ in $E^{n}$ is an irreducible group generated by reflections in the hyperplanes of symmetry of $I I$ and that every p.p. of $\Pi$ (for definition, see $[4$, p. 605]) can be obtained by taking every $n$th vertex of a p.p. of the spherical honeycomb corresponding to $\$ 3[4$, p. 605]. Hence by 4.2 we have:

5.3. Corollary to 4.2. If a Petrie polygon of a regular polytope $\Pi$ in $E^{n}$ has $h$ vertices, then the number of hyperplanes of symmetry of $\Pi$ is $n h / 2$.

6. Roots. We leave the spherical honeycomb and introduce two nonzero normal vectors for each r.h. in such a way that each element of $\$(5)$ permutes the resulting $n h$ vectors. These vectors will be called roots. Thus if $\rho$ is a root, so is $-\rho$. The $n$ roots lying along the inwardly directed normals to a fixed fundamental chamber $F$ are called fundamental roots. Since no r.h. has points interior to $F$, every root $\rho$ is a linear combination of fundamental roots in which all coefficients are non-negative or all are nonpositive; in the first (second) case $\rho$ is called positive (negative) and we write $\rho>0(\rho<0)$. From the equation for the reflection $R$ in the hyperplane orthogonal to $\rho$,

$$
R \eta=\eta-2 \rho(\eta, \rho) /(\rho, \rho),
$$

we get the following result, important for our purposes [11, p. 19-01]:

6.2. Lemma. The reflection in the hyperplane orthogonal to a fundamental root $\alpha$ maps $\alpha$ upon $-\alpha$ and permutes the remaining positive roots.

For roots a somewhat sharper analogue of 4.6 exists.

6.3. Theorem. Let the fundamental roots be ordered similarly to 3.1 so that $\alpha_{1}, \alpha_{2}, \cdots, \alpha_{s}$ are mutually orthogonal as also are $\alpha_{s+1}, \cdots, \alpha_{n}$. Let $R_{j}$ be the reflection in the wall $W_{j}$ orthogonal to $\alpha_{j}$. Let $\alpha_{k}=\alpha_{j}$ and $R_{k}=R_{j}$ if $k \equiv j(\bmod n)$, and then let $\rho_{k}=R_{1} R_{2} \cdots R_{k-1} \alpha_{k}, k=1,2, \cdots$ Then (1) the positive roots are the $\rho$ 's given by $k=1,2, \cdots, n h / 2 ;(2)$ the negative roots are given by $k=n h / 2+1, \cdots, n h$.

Proof. Suppose that $1 \leqq k \leqq n h / 2$. Then if $1 \leqq j<k$, it follows by 4.6 that $\rho_{k} \neq \pm \rho_{j}$, so that $R_{j-1} R_{j-2} \cdots R_{1} \rho_{k} \neq \pm \alpha_{j}$. Thus by 6.2 the roots $R_{j-1} R_{j-2} \cdots$ $R_{1} \rho_{k}$ and $R_{j} R_{j-1} \cdots R_{1} \rho_{k}$ have the same sign. Taking $j=1,2, \cdots, k-1$, we see that $\rho_{k}$ has the same sign as $R_{k-1} \cdots R_{1} \rho_{k}=\alpha_{k}$ and hence is positive. Sup- 
pose next that $n h / 2<k \leqq n h$. Since $R_{1} R_{2} \cdots R_{n h}=1$, it now follows that $\rho_{k}=-R_{n h} R_{n h-1} \cdots R_{k+1} \alpha_{k}$; applying (1) to the case in which the $\alpha$ 's and $R$ 's are relabeled so as to appear in reverse order, we get (2).

There exists the result that all roots are given by $\rho_{k}, k=1,2, \cdots, n h$, for an arbitrary initial ordering of the fundamental roots. However 4.2 is valid for all initial orderings if and only if (s) contains the central inversion. These statements can be proved by modifications of the methods already introduced.

By a simple computation using the definition of the $\rho$ 's and 6.1 , one can also prove the recursion formula

$$
\rho_{k}=\alpha_{k}+\sum_{j=1}^{k-1} a_{k j} \rho_{j}, \quad a_{k j}=-2\left(\alpha_{k}, \alpha_{j}\right) /\left(\alpha_{j}, \alpha_{j}\right) .
$$

6.5. Corollary. Suppose that the fundamental roots are partitioned under the action of \& into transitive sets of $n_{1}, n_{2} . \cdots, n_{r}$ elements. Then the set of all roots is partitioned into transitive sets of $n_{1} h, n_{2} h, \cdots, n_{r} h$ elements.

Proof. This is clear from 6.2.

6.6. Corollary. If $h$ is even, the set of all roots can be partitioned into $h$ fundamental sets, each consisting of the roots which lie along the inwardly directed normals to the walls of a fundamental chamber.

Proof. $R_{1} R_{2} \cdots R_{s}\left(\alpha_{1}, \alpha_{2}, \cdots, \alpha_{n}\right)=\left(-\rho_{1},-\rho_{2}, \cdots,-\rho_{s}, \rho_{s+1}, \cdots, \rho_{n}\right)$ is a fundamental set. By 6.3 one can apply $1, R, \cdots, R^{h / 2-1}$ in turn to this set to get $h / 2$ fundamental sets of which the union contains exactly one of $\rho$ and $-\rho$ for each root $\rho$. Hence one gets the required result by adjoining to the sets already obtained their negatives.

Consulting the known list of values of $h[4$, p. 618], one sees that $h$ is odd only if $B=\mathfrak{A}_{h-1}$, the symmetry group of the regular $(h-1)$-simplex. Representing (3) by the permutations of the $h$ vertices of the simplex, one sees that the question left open by 6.6 can be phrased as follows:

6.7. If $h$ is odd, is it possible to order the numbers $1,2, \cdots, h$ in $h$ ways so that the resulting $h(h-1)$ ordered consecutive pairs are distinct?

If $h=3$ or 5 , the answer is no, but the general question seems to be open.

7. Traces. Here we prove two lemmas to be used in the proof of 1.2.

Recall that $\alpha_{1}, \alpha_{2}, \cdots, \alpha_{n}$ denote the fundamental roots relative to a fixed fundamental chamber $F$. If $\mu=\sum_{1}^{n} x^{j} \alpha_{j}$ is an arbitrary vector, we set $\sum x^{i}=\operatorname{tr} \mu$, the trace of $\mu$.

7.1. Lemma. If $\mu$ is an arbitrary vector, then $\operatorname{tr} \mu=\sum(\mu, \rho) /(\rho, \rho)$, the sum being on the positive roots $\rho$.

Proof. Set $\eta=\sum \rho /(\rho, \rho)$. The reflection in the hyperplane orthogonal to a fundamental root $\alpha$ maps $\eta$ onto $\eta-2 \alpha(\eta, \alpha) /(\alpha, \alpha)$ by 6.1 . By 6.2 this last 
vector is equal to $\eta-2 \alpha /(\alpha, \alpha)$. Hence $(\eta, \alpha)=1=\operatorname{tr} \alpha$, for each fundamental $\alpha$. It follows by linearity that for an arbitrary $\mu$ we have $\operatorname{tr} \mu=(\eta, \mu)$, that is 7.1. The argument used here occurs in $[11$, p. 19-01] in a similar context.

7.2. Lemma. If $\mu$ is an arbitrary vector, and if $k$ is the number of r.h. in (5), then

$$
\sum_{\rho>0}(\mu, \rho)^{2} /(\mu, \mu)(\rho, \rho)=k / n .
$$

Proof. $Q(\mu)=\sum(\mu, \rho)^{2} /(\rho, \rho)$ and $(\mu, \mu)$ are quadratic forms in $\mu$ which are invariant under (f). Since (F) is algebraically irreducible over the reals, $Q(\mu)=c(\mu, \mu)$ for some real $c$. Letting $\mu$ run through an orthonormal basis of $E^{n}$, one gets $c=k / n$, as required.

8. Crystallographic restriction, dominant root. Assume that $\overline{(3)}$ is an infinite irreducible discrete g.g.r. on $E^{n}$, that 0 is a "special point" which lies on one member of each family of parallel r.h. of $\bar{G}$, and that (B) is the subgroup of $\bar{\xi}$ leaving 0 fixed (see [4]).

8.1. Lemma. Under the assumptions of the preceding paragraph, the roots corresponding to $\&$ can be so chosen that $2(\rho, \sigma) /(\sigma, \sigma)$ is an integer for each pair of roots $\rho, \sigma$.

Proof. Because $\overline{(3)}$ is irreducible, it easily follows that each r.h. through 0 has other r.h. parallel to it. Now choose each root $\rho$ to be equal in length to the distance between consecutive r.h. orthogonal to $\rho$. Let $\rho$ and $\sigma$ be any two roots. The point $2 \rho$ is a transform of 0 by $\overline{(5)}$ and so lies on a r.h. orthogonal to $\sigma$. Since the distance of this r.h. from 0 is $2(\rho, \sigma) /(\sigma, \sigma)^{1 / 2}$, we conclude that the latter number is an integral multiple of the length $(\sigma, \sigma)^{1 / 2}$ of $\sigma$, as desired [7, p. 404].

Henceforth we impose these crystallographic restrictions on $B$ and the root system. It is in this case that there exists a corresponding Lie group which is closely related through its algebraic and topological properties with $\overline{\mathfrak{G}}$, (5) and the root system $[1 ; 2 ; 3 ; 13]$.

From $6.1,6.3$ and 8.1 , it follows that each root has integral coefficients in terms of the fundamental roots. One can also easily conclude from 8.1 the following fact:

8.2. Lemma. If $\rho$ and $\sigma$ are two roots such that $\rho \neq \pm \sigma$ and $(\rho, \rho) \leqq(\sigma, \sigma)$, then $2(\sigma, \rho) /(\sigma, \sigma)=0,1$ or -1 .

Cartan [3, p. 256] has proved the existence of a dominant root $\mu=\sum y^{j} \alpha_{j}$ with the property that, if $\rho=\sum x^{j} \alpha_{j}$ is any root, then $y^{j} \geqq x^{j}$ for all $j$. It follows that $\mu$ is in $F$, or, equivalently, that $\left(\mu, \alpha_{j}\right) \geqq 0$ for each fundamental root $\alpha_{j}$, since otherwise one could increase a coefficient of $\mu$ by reflection in some wall of $F$.

8.3. LemMa. If $\mu$ is the dominant root and $\rho$ is any root, then $(\rho, \rho) \leqq(\mu, \mu)$. 
Proof. Since there is an element of $B$ mapping $\rho$ onto a root in $F$ of the same length, we may assume that $\rho$ is in $F$. Since $\mu-\rho$ has only non-negative coefficients, and $\rho$ and $\mu$ are in $F$, it follows that $(\mu-\rho, \mu) \geqq 0$ and $(\rho, \mu-\rho) \geqq 0$. Thus $(\mu, \mu) \geqq(\rho, \mu) \geqq(\rho, \rho)$, with equality only if $\mu=\rho$.

The stage is now set for the proof of the second main theorem.

8.4. Theorem. If $\mu$ is the dominant root, then the number of r.h. is $k$ $=n(1+\operatorname{tr} \mu) / 2$.

Proof. If $\rho>0$, then $(\mu, \rho) \geqq 0$, and then 8.2 and 8.3 imply that $2(\mu, \rho) /(\mu, \mu)$ $=0$ or 1 if $\rho \neq \mu$. Hence using 7.1 and 7.2 and noting that the range of summation below is the set of positive roots, we have

$$
\begin{aligned}
\operatorname{tr} \mu & =\sum(\mu, \rho) /(\rho, \rho)=\sum(2(\mu, \rho) /(\mu, \mu))((\mu, \mu) / 2(\rho, \rho)) \\
& =\sum(2(\mu, \rho) /(\mu, \mu))^{2}((\mu, \mu) / 2(\rho, \rho))-1 \\
& =2 \sum(\mu, \rho)^{2} /(\mu, \mu)(\rho, \rho)-1=2 k / n-1 .
\end{aligned}
$$

A similar computation also explains an analogous formula encountered by Coxeter in his work on extreme quadratic forms [7, p. 413].

9. Trace distribution. Following is another easily verified property of root systems which the author has not been able to explain in general terms. By 4.3 the characteristic values of $R$ are of the form $\exp \left(2 \pi i m_{j} / h\right)$ with 1 $=m_{1} \leqq m_{2} \leqq \cdots \leqq m_{n}=h-1$. The numbers $m_{j}$ have been called the $e x$ ponents of $B$, and enter into many questions concerning $(5 ;$; for example, the numbers $m_{j}+1$ are the degrees of a basic set of invariants for $(B)$, and, if $B$ is crystallographically restricted, then the Poincaré polynomial of the corresponding Lie group is $\mathrm{II}\left(1+t^{2 m_{j}+1}\right)$ (see [12] for other properties). Under this restriction one can also prove by verification the following curious fact.

9.1. Let the number of positive roots of trace $1,2, \cdots, h-1$ be $p_{1}, p_{2}, \cdots$, $p_{h-1}$, respectively. Then $p_{1} \geqq p_{2} \geqq \cdots \geqq p_{h-1}$ and the partition conjugate to that determined by the p's consists of the $m$ 's, the exponents of $(\$)$.

For example, if $(5)=\mathfrak{D}_{4}$ (in the usual notation), then the roots of trace $1,2,3,4,5$ occur with multiplicities of $4,3,3,1,1$, respectively. The partition of 12 conjugate to the last list of numbers consists of the numbers $1,3,3,5$, the exponents of $\mathfrak{I}_{4}$.

\section{REFERENCES}

1. E. Cartan, Thèse, Paris, Nony, 1894. 256.

2. — La géométrie des groupes simples, Annali Mat. Pura Appl. vol. 4 (1927) pp. 209-

3. - Complément au mémoire "sur la géométrie des groupes simples," Annali Mat. Pura Appl. vol. 5 (1928) pp. 253-260.

4. H. S. M. Coxeter, Discrete groups generated by reflections, Ann. of Math. vol. 35 (1934) pp. $588-621$.

5. — Lösung der Aufgabe 245, Jber. Deutsch. Math. Verein. vol. 49 (1939) pp. 4-6.

6. —, Regular polytopes, New York, 1949. 
7. —, Extreme forms, Canad. J. Math. vol. 3 (1951) pp. 391-441.

8. - The product of the generators of a finite group generated by reflections, Duke Math. J. vol. 18 (1951) pp. 765-782.

9. G. Frobenius, Über Matrizen aus positiven Elementen, Preuss. Akad. Wiss. Sitzungsber. (1908) pp. 471-476.

10. D. König, Theorie der endlichen und unendlichen Graphen, New York, 1950.

11. Séminaire "Sophus Lie," Paris, 1954-1955.

12. G. C. Shepard, Some problems on finite reflection groups, Enseignement Math. vol. 2 (1956) pp. 42-48.

13. H. Weyl, Über die Darstellungen halbeinfacher Gruppen durch lineare Transformationen. Math. Z. vol. 23 (1925) pp. 271-309, vol. 24 (1926) pp. 328-395

\section{University of California,}

Los Angeles, Calif. 